\title{
Plasma free fatty acid and catecholamine levels in patients with acute myocardial infarction
}

\author{
W. Januszewicz, M. Sznajderman, M. Ciświcka-Sznajderman, B. Wocial, \\ and Z. Rymaszewski \\ From the Second Department of Medicine, Academy of Medicine, \\ 59 Nowogrodzka Street, Warsaw, Poland
}

In 25 men with acute myocardial infarction simultaneous determinations of free fatty acid $(F F A)$, noradrenaline, and adrenaline were made in blood samples obtained on the Ist and the Ioth day after the acute attack. In the acute phase of myocardial infarction the levels of FFA and noradrenaline were significantly higher than on the Ioth day of illness and revealed a highly significant positive correlation. The blood adrenaline levels were not significantly raised and did not correlate with the FFA levels. Probable mechanisms and significance of metabolic changes occurring in the acute phase of myocardial infarction are discussed.

Acute myocardial infarction is accompanied by profound metabolic changes. It is now well established that the activity of the sympathoadrenal system is increased in acute myocardial infarction, as reflected in augmented urinary excretion of noradrenaline and adrenaline and raised plasma levels of these catecholamines (Januszewicz et al., 1968; Klein et al., 1968; McDonald et al., 1969; Valori, Thomas, and Shillingford, 1967a, b). It was also shown that the excretion of catecholamines might vary in relation to the severity of the clinical course and to the nature of concomitant complications (Januszewicz et al., 1968; Jewitt et al., 1969; Valori et al., 1967a, b). Recently, attention has been paid to the increased plasma free fatty acids (FFA) in patients with acute myocardial infarction (Gupta et al., 1969; Kurien and Oliver, 1966; Oliver, Kurien, and Greenwood, I968). The observations on the higher incidence of cardiac arrhythmias and sudden death in patients with myocardial infarction exhibiting strikingly raised serum FFA are particularly noteworthy (Gupta et al., 1969; Oliver et al., 1968; Oliver, 1968). Other authors have not found a relation between the initial rise of plasma FFA and the subsequent clinical course of acute myocardial infarction; however, they have noted a repeated increase of plasma FFA coinciding with the onset of complications (Rutenberg, Pamintuan, and Soloff, 1969). It seems probable that the increase of plasma FFA under

Received 16 November 1970. these circumstances is related to augmented secretion of catecholamines. The present study was undertaken to investigate a possible relation between the increase of plasma FFA and that of plasma catecholamines in acute myocardial infarction.

\section{Patients and methods}

Twenty-five men with acute myocardial infarction, consecutively admitted to the coronary care unit, were studied. The age range was 35-65 years, with a mean of $5 \mathrm{r} \cdot 4$ years. The diagnosis of myocardial infarction was based on the clinical picture, typical electrocardiographic findings, and raised enzyme levels. None of the patients received heparin, corticosteroids, clofibrate, glucose infusions, or other drugs which might have influenced the parameters studied. None of the patients had a history of diabetes mellitus. The patients were continuously monitored with an oscilloscope, with frequent pulse and blood pressure measurements. The venous blood samples of about $25 \mathrm{ml}$ were obtained within 12 hours from the onset of the acute attack, at least 5 hours after a meal. The infarction was transmural in 22 patients and subendocardial in 3 patients. The localization of the infarction was anterior in 8 patients, anterolateral and inferolateral in 2 patients each, and inferior in 8 patients. None of the patients had evidence of shock. Four patients gave a history of hypertension. In 4 patients the systolic blood pressure was $160 \mathrm{mmHg}$ or higher, and in ro patients the diastolic blood pressure equalled or exceeded $95 \mathrm{mmHg}$ at the time of blood collection.

Various degrees of heart failure were found in 18 patients; in 12 of them the symptoms of heart 

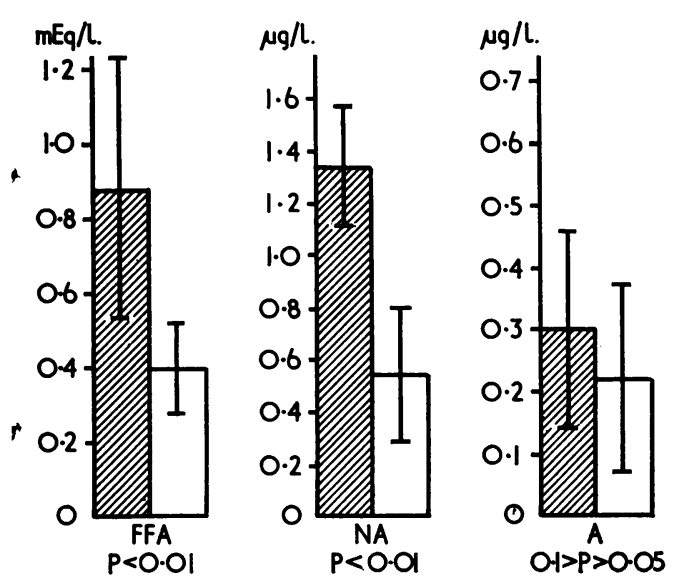

FIG. Mean values of free fatty acids (FFA), noradrenaline $(N A)$, and adrenaline $(A)$.

Shaded blocks represent the Ist day and open blocks the Ioth day of observation. Vertical lines represent standard deviation.

failure subsided within a few days. One patient y was admitted with intractable pulmonary oedema. Cardiac arrhythmias were found in 13 patients; in 4 patients they were multiple. There were 2 cases of atrial fibrillation and I case of ventricular fibrillation successfully treated by direct current countershock. Four patients had ventricular ectopic beats of a frequency exceeding I: IO. Three patients were found to have Ist or and

- degree atrioventricular block which, in 2 of them, increased periodically to complete atrioventricular block. Out of the 25 patients studied, 4 died. In I case death was due to cardiac rupture within the first 24 hours, in 2 cases to intractable pulmonary oedema; one patient died with symptoms of septicaemia. In 23 patients control blood samples were obtained ro days after the onset of acute myocardial infarction. At the time of the control blood collection, none of the patients ex-

- perienced angina; heart failure persisted in 2 patients and arrhythmia was still present in 2 patients who had infrequent ventricular and supraventricular ectopic beats.

Plasma noradrenaline (recovery $85 \pm 2.9 \%$ ) and adrenaline (recovery $8 \mathrm{I} \pm \mathrm{I} \cdot 7 \%$ ) were determined fluorometrically by the method of Vendsalu (1960). $18 \mathrm{ml}$ blood were collected into a siliconized test-tube containing $2 \mathrm{ml}$ I per cent EDTA with the addition of a small amount of metabisulphite sodium. After the blood had been deproteinized by the addition of $0.4 \mathrm{~N}$ perchloric acid, it was centrifuged for 10 minutes at $10^{\circ} \mathrm{C}$. The catecholamines were then isolated from the sample by means of column chromatography on Dowex-X 4200 mesh. After the column had been 1. activated, the catecholamines were eluted with I $\mathrm{N}$ hydrochloric acid. The quantitative estimation of noradrenaline and adrenaline in the eluate was performed with the trihydroxyl method of
Euler and Lishajko (I96r). The normal plasma concentrations of noradrenaline and adrenaline accerding to this laboratory are $0.67 \pm 0.3 \mu \mathrm{g} / \mathrm{l}$. and $0.22 \pm 0.09 \mu \mathrm{g} / \mathrm{l}$., respectively. Plasma FFA concentration was determined by the method of Dole (1956). The results were subjected to statistical analysis; the differences were considered statistically significant when the $P$ index was less than 0.05 . Mean values are followed by \pm which mean standard deviation.

\section{Results}

The plasma FFA levels in the first 24 hours of acute myocardial infarction ranged from 0.225 to $2.303 \mathrm{mEq} / \mathrm{l}$, with the mean of $0.874 \pm$ $0.343 \mathrm{mEq} / \mathrm{l}$. The simultaneously measured plasma levels of noradrenaline ranged from 0.52 to $2.80 \mu \mathrm{g} / \mathrm{l}$., with the mean of $\mathrm{r} \cdot 34 \pm 0.22$ $\mu \mathrm{g} / \mathrm{l}$. and those of adrenaline ranged from 0.12 to $0.95 \mu \mathrm{g} / 1$., with the mean of $0.30 \pm 0.16 \mu \mathrm{g} / 1$.

The corresponding control values, found ro days after the onset of infarction, were: FFA: $0.208-0.726$, mean $0.387 \pm 0.122$ $\mathrm{mEq} / 1$; noradrenaline: $0.20-0.99$, mean 0.53 $\pm 0.26 \mu \mathrm{g} / 1$. ; adrenaline: $0.10-0.90$, mean $0.22 \pm 0.15 \mu \mathrm{g} / \mathrm{l}$.

The statistical analysis revealed that the differences between the first and the tenth day of myocardial infarction were significant for noradrenaline $(P<0.01)$, and FFA $(P<$ 0.01 ); the difference in plasma adrenaline was not statistically significant $(P>0.05)$ (Fig.). A highly significant positive correlation was found between the plasma concentrations of FFA and noradrenaline in the early acute stage of myocardial infarction $(r=+0.74 \mathrm{I}$; $P<0.01)$. The plasma FFA and adrenaline did not correlate $(r=+0.010 ; P>0 \cdot r)$.

\section{Discussion}

In accordance with the reports of other authors (Gupta et al., 1969; Kurien and Oliver, 1966; Oliver, 1968; Rutenberg et al., 1969), our study showed a significant increase in plasma FFA in the first 24 hours of acute myocardial infarction with subsequent normalization on the roth day. The difference between the first and the tenth day was statistically highly significant. Similarly, the plasma noradrenaline concentration was significantly raised on the Ist day in comparison with the Ioth day. The highly significant correlation between the concentrations of noradrenaline and FFA, found in the acute stage of myocardial infarction, and the parallel decline of these values in time deserve particular attention. On the other hand, the plasma adrenaline concentration increased slightly and did not correlate with plasma FFA. 
The mechanism and the significance of the increase of plasma FFA concentration in acute myocardial infarction has been a matter of interest, especially so in connexion with the role of FFA in the myocardial metabolism. The increased circulating plasma FFA promote augmented myocardial oxygen consumption which may be particularly harmful for ischaemic myocardium. A relation between the post-heparin increase of plasma FFA and the incidence of cardiac arrhythmias was reported in dogs with acute coronary artery occlusion (Kurien, Yates, and Oliver, 1969). It was also shown that in experimental conditions the FFA diminish the contractility of the hypoxic heart muscle (Henderson, Most, and Sonnenblick, 1969). Several recent clinical observations suggest that free fatty acids may also play an important role in the clinical course of acute myocardial infarction. In the present study we were particularly concerned with the relation between plasma FFA and catecholamine concentrations in acute myocardial infarction. In a previous report (Januszewicz et al., 1968) we were able to show an augmented urinary excretion of catecholamines in acute myocardial infarction and the relation between the excretion of respective catecholamines and the severity of the clinical course and the nature of complications. Estimation of plasma catecholamine concentrations is technically more complicated than that of urinary excretion, and it does not reflect diurnal variations of the sympatho-adrenal system. However, better insight into the existing interrelations and directions of changes can be gained when several parameters are measured in the same blood sample.

It is known that noradrenaline is one of the most potent factors releasing FFA into the blood through the activation of tissue lipase and the subsequent hydrolysis of triglycerides. The highly significant correlation between the increased levels of FFA and noradrenaline which we have been able to show in the acute stage of myocardial infarction, as well as the subsequent parallel decrease of these levels, justifies the suggestion that the raised plasma FFA in acute myocardial infarction may be related to the increased noradrenaline production caused by the augmented activity of the sympathoadrenal system in response to the infarction.

The assessment of the relation between plasma FFA and catecholamine concentration and the clinical course of acute myocardial infarction is beyond the scope of this investi- gation. Further studies are in progress to get information on ultimate prognosis in acute myocardial infarction in relation to the magnitude of metabolic changes in the acute phase of the disease.

\section{References}

Dole, V. P. (1956). A relation between non-esterified fatty acids in plasma and the metabolism of glucosae. Fournal of Clinical Investigation, 35, 150.

Euler, U. S. von, and Lishajko, R. (I96I). Improved technique for the fluorometric estimation of catecholamines. Acta Physiologica Scandinavica, $51,348$.

Gupta, D. K., Young, R., Jewitt, D. E., Hartog, M., and Opie, L. H. (1969). Increased plasma-freefatty-acid concentrations and their significance in patients with acute myocardial infarction. Lancet, 2, 1209.

Henderson, A. H., Most, A. S., and Sonnenblick, E. H. (1969). Depression of contractility in rat heart muscle by free fatty acids during hypoxia. Lancet, 2, 825 .

Januszewicz, W., Sznajderman, M., Wocial, B., and Preibisz, J. (1968). Urinary excretion of free norepinephrine and free epinephrine in patients with acute myocardial infarction in relation to its clinical course. American Heart fournal, 76, 345.

Jewitt, D. E., Mercer, C. J., Reid, D., Valori, C., Thomas, M., and Shillingford, J. P. (1969). Free noradrenaline and adrenaline excretion in relation to the development of cardiac arrhythmias and heart-failure in patients with acute myocardial infarction. Lancet, I, 635.

Klein, R. F., Troyer, W. G., Thompson, H. K., Bogdonoff, M. D., and Wallace, A. G. (1968). Catecholamine excretion in myocardial infarction. Archives of Internal Medicine, 122, 476.

Kurien, V. A., and Oliver, M. F. (1966). Serum-freefatty-acids after acute myocardial infarction and cerebral vascular occlusion. Lancet, 2, I22.

Kurien, V. A., Yates, P. A., and Oliver, M. F. (1969). Free fatty acids, heparin, and arrhythmias during experimental myocardial infarction. Lancet, 2, 185 .

McDonald, L., Baker, C., Bray, C., McDonald, A., and Restieaux, N. (1969). Plasma-catecholamines after cardiac infarction. Lancet, 2, 102I.

Oliver, $M$. (1968). The relationship of serum free fatty acids to arrhythmias and death. In Acute Myocardial Infarction, p. 25I. Ed. by D. G. Julian and M. F. Oliver. Livingstone, Edinburgh.

Oliver, M. F., Kurien, V. A., and Greenwood, T. W. (I968). Relation between serum-free-fatty-acids and arrhythmias and death after acute myocardial infarction. Lancet, $1,710$.

Rutenberg, H. L., Pamintuan, J. C., and Soloff, L. A. (1969). Serum-free-fatty-acids and their relation to complications after acute myocardial infarction. Lancet, 2, 559.

Valori, C., Thomas, M., and Shillingford, J. P. (I967a). Urinary excretion of free noradrenaline and adrenaline following acute myocardial infarction. Lancet, I, 127.

Valori, C., Thomas, M., and Shillingford, J. (1967b). Free noradrenaline and adrenaline excretion in relation to clinical syndromes following myocardial infarction. American fournal of Cardiology, 20, 605.

Vendsalu, A. (1960). Studies on adrenaline and noradrenaline in human plasma. Acta Physiologica Scandinavica, 49, Suppl. 173, p. 23. 